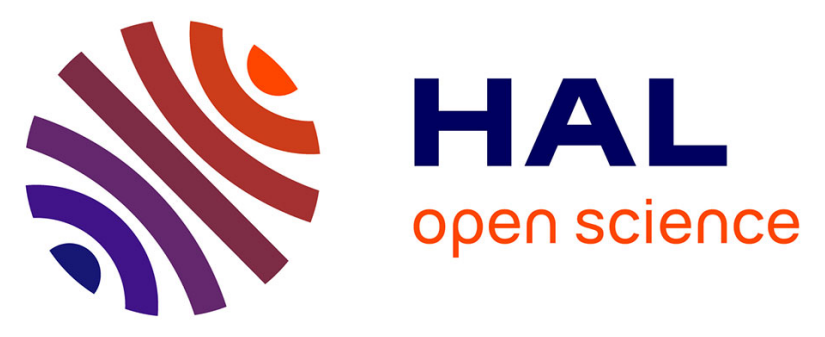

\title{
Determination of the Distributions of the Spin-Hamiltonian Parameters in Spin Triangles: A Combined Magnetic Susceptometry and Electron Paramagnetic Resonance Spectroscopic Study of the Highly Symmetric $[\mathrm{Cr} 3 \mathrm{O}(\mathrm{PhCOO}) 6(\mathrm{py}) 3](\mathrm{ClO}) \times 0.5 p y$
}

Athanassios K. Boudalis, Guillaume Rogez, Philippe Turek

\section{To cite this version:}

Athanassios K. Boudalis, Guillaume Rogez, Philippe Turek. Determination of the Distributions of the Spin-Hamiltonian Parameters in Spin Triangles: A Combined Magnetic Susceptometry and Electron Paramagnetic Resonance Spectroscopic Study of the Highly Symmetric $[\mathrm{Cr} 3 \mathrm{O}(\mathrm{PhCOO}) 6($ py $) 3](\mathrm{ClO}) \times 0.5$ py. Inorganic Chemistry, 2018, 10.1021/acs.inorgchem.8b01764 . hal-01985440

\author{
HAL Id: hal-01985440 \\ https://hal.science/hal-01985440
}

Submitted on 25 Jan 2019

HAL is a multi-disciplinary open access archive for the deposit and dissemination of scientific research documents, whether they are published or not. The documents may come from teaching and research institutions in France or abroad, or from public or private research centers.
L'archive ouverte pluridisciplinaire HAL, est destinée au dépôt et à la diffusion de documents scientifiques de niveau recherche, publiés ou non, émanant des établissements d'enseignement et de recherche français ou étrangers, des laboratoires publics ou privés. 


\section{Determination of the distributions of the spin}

\section{Hamiltonian parameters in spin triangles: a}

\section{combined magnetic susceptometry and EPR}

spectroscopic study of the highly-symmetric

\section{$\left[\mathrm{Cr}_{3} \mathrm{O}(\mathrm{PhCOO})_{6}(\mathrm{py})_{3}\right]\left(\mathrm{ClO}_{4}\right) \cdot 0.5 \mathrm{py}$}

Athanassios K. Boudalis ${ }^{a}$, Guillaume Rogez ${ }^{b}$, Philippe Turek $^{a}$

anstitut de Chimie de Strasbourg (UMR 7177, CNRS-Unistra), Université de Strasbourg, 4 rue Blaise Pascal, CS 90032, F-67081 Strasbourg, France. E-mail: bountalis@unistra.fr.

${ }^{\text {b}}$ Université de Strasbourg, CNRS, Institut de Physique et Chimie des Matériaux de Strasbourg (IPCMS), UMR 7504, F-67000 Strasbourg, France.

Keywords: magnetic properties, EPR spectroscopy, spin triangles, Dzyaloshinskii-Moriya interactions, distributions

\section{Corresponding Author}

*Email: bountalis@unistra.fr 


\section{Abstract}

Magnetic susceptibility and X-band Electron Paramagnetic Resonance (EPR) studies have been carried out on the highly symmetric $\left[\mathrm{Cr}_{3} \mathrm{O}(\mathrm{PhCOO})_{6}(\mathrm{py})_{3}\right]\left(\mathrm{ClO}_{4}\right) \cdot 0.5 \mathrm{py}(\mathbf{1})$, whose cation exhibits a $D_{3 h}$ crystallographically-imposed molecular symmetry. While magnetic susceptibility data can be interpreted with an equilateral magnetic model described by the effective multispin Hamiltonian $\hat{H}=-2 J\left(\hat{\mathbf{S}}_{1} \cdot \hat{\mathbf{S}}_{2}+\hat{\mathbf{S}}_{2} \cdot \hat{\mathbf{S}}_{3}+\hat{\mathbf{S}}_{3} \cdot \hat{\mathbf{S}}_{1}\right)$, EPR data require an isosceles model described by the multispin Hamiltonian $\hat{H}=-2 J\left(\hat{\mathbf{S}}_{1} \cdot \hat{\mathbf{S}}_{2}+\hat{\mathbf{S}}_{2} \cdot \hat{\mathbf{S}}_{3}\right)-2 J^{\prime} \hat{\mathbf{S}}_{3} \cdot \hat{\mathbf{S}}_{1}$, where $\Delta J=J-J^{\prime} \neq 0$. Moreover, EPR data reveal the interplay of antisymmetric exchange (or Dzyaloshinskii-Moriya) interactions, described by a $2 \mathbf{G}\left(\hat{\mathbf{S}}_{1} \times \hat{\mathbf{S}}_{2}+\hat{\mathbf{S}}_{2} \times \hat{\mathbf{S}}_{3}+\hat{\mathbf{S}}_{3} \times \hat{\mathbf{S}}_{1}\right)$ term, which induce significant anisotropy to the $S_{\mathrm{T}}=1 / 2$ ground state of $\mathbf{1}$, as well as an important broadening of the $g_{\perp}$ resonance ( $g$-strain). Through careful analysis of these data, and in conjunction with neutron scattering data, this $g$-strain can be deconvoluted into distributions of the individual spin Hamiltonian parameters $\Delta J$ and $|\mathbf{G}|$. This method of analysis provides simultaneous estimates of the central values and distribution profiles of the spin Hamiltonian parameters, which are shown not to be described by monodisperse values. 


\section{Introduction}

Antiferromagnetic triangular complexes of half-integer spins, or "spin triangles", were the first molecular clusters to be studied with respect to their magnetic properties and the first to be treated in a quantum mechanically coherent manner by employing a multispin (or 'microscopic' spin) Hamiltonian. ${ }^{1,2}$ This was a very early adoption of the use of effective spin Hamiltonians, which instead of using crystal field parameters, more convenient for optical spectroscopy, made use of effective parameters more convenient for magnetic and EPR studies (for a rigorous and subtle analysis of the concepts surrounding spin Hamiltonians see the review of Rudowicz and Karbowiak $^{3}$ ). Those triangles are very relevant to the intense current research on Quantum Spin Liquids ${ }^{4}$ because they can be considered as the elementary units of extended systems like triangular and Kagome geometrically frustrated antiferromagnets, which are predicted to attain this non-ordered magnetic state of matter. The study of isolated antiferromagnetic half-integer triangles and the design of perfectly frustrated such triangles ${ }^{5}$ can help in the understanding of the magnetism of their extended congeners and the design of ideal materials like "perfect Kagome antiferromagnets”.,

More recently, spin triangles have become of interest in the context of the use of molecular nanomagnets (MNMs) as spin qubits for Quantum Information Processing (QIP). ${ }^{8}$ One such scheme proposes the use of the ground-doublet Zeeman levels of antiferromagnetic half-integer rings as the two qubit states. ${ }^{9}$ It was then demonstrated experimentally ${ }^{10-12}$ and theoretically ${ }^{13}$ that such a scheme could be implemented by spin triangles, which constitute the smallest possible rings; under this scheme, the two qubit states correspond to the two possible spin projections of the ground state doublet $\left(M_{S}= \pm 1 / 2\right)$. It was then proposed that instead of this scheme, the same molecules could be used as spin qubits, by using employing their spin chirality 
as the computational degree of freedom, which would allow electric manipulation ${ }^{14,15}$ and longer coherence times, ${ }^{16}$ a proposition recently validated by us through the observation of magnetoelectric coupling in complex $\left[\mathrm{Fe}_{3} \mathrm{O}(\mathrm{PhCOO})_{6}(\mathrm{py})_{3}\right]\left(\mathrm{ClO}_{4}\right) \cdot \mathrm{py}^{17}$

As pointed out by Di Vincenzo in his famous checklist, ${ }^{18}$ in order to employ a system for a qubit implementation, we need a precise description of its spin Hamiltonian. Indeed, the gross picture for spin triangles has been relatively well elucidated for some time now: ${ }^{19}$ for an equilateral magnetic system in which only isotropic exchange interactions occur, according to the $\hat{H}=-2 J\left(\hat{\mathbf{S}}_{1} \cdot \hat{\mathbf{S}}_{2}+\hat{\mathbf{S}}_{2} \cdot \hat{\mathbf{S}}_{3}+\hat{\mathbf{S}}_{3} \cdot \hat{\mathbf{S}}_{1}\right)$ multispin Hamiltonian, the ground state is a pair of degenerate doublets, followed by an excited quartet; in the above convention, $J<0$ implies antiferromagnetic interactions, whereas the Hamiltonian formalism implies that all isotropic exchange couplings have the same sign and strength. In practice, an abundance of experimental data has shown that the degeneracy is invariably lifted. Initially, this had been attributed to a symmetry lowering of the Heisenberg-Dirac-van Vleck (HDVV) isotropic Hamiltonian, also known as "Magnetic Jahn-Teller Effect", leading to a $\hat{H}=-2 J\left(\hat{\mathbf{S}}_{1} \cdot \hat{\mathbf{S}}_{2}+\hat{\mathbf{S}}_{2} \cdot \hat{\mathbf{S}}_{3}\right)-2 J$ ' $\hat{\mathbf{S}}_{3} \cdot \hat{\mathbf{S}}_{1}$ multispin Hamiltonian, with a magnetic asymmetry $\Delta J=J-J^{\prime} ;{ }^{20}$ this form implies that whereas the isotropic exchange couplings $J$ and $J$ ' have the same sign, they are of different sizes.

Alternative attempts were made by Uryû and Friedberg ${ }^{21}$ to attribute this lifting of degeneracy to single-ion zero-field splitting (zfs), but these demonstrated a negligible effect of that parameter to the reproduction of magnetic susceptibility data, a conclusion recently reached by Boča. ${ }^{22}$ Instead, consideration of magnetic susceptibility and magnetic heat capacity data by Mishima and Uryû demonstrated the ability of antisymmetric exchange (or DzyaloshinskiiMoriya) terms $2 \mathbf{G}\left(\hat{\mathbf{S}}_{1} \times \hat{\mathbf{S}}_{2}+\hat{\mathbf{S}}_{2} \times \hat{\mathbf{S}}_{3}+\hat{\mathbf{S}}_{3} \times \hat{\mathbf{S}}_{1}\right)$ to better account for the low-temperature behavior 
of these complexes, without the need to assume such a symmetry lowering. ${ }^{23}$ Analysis of EPR data of basic iron(III) and chromium(III) carboxylates reconciled those competing views; it was shown that the axial spectra of these complexes could only be explained by the assumption of antisymmetric exchange interactions acting in tandem with a low magnetic symmetry. ${ }^{24,25}$ Such terms need to be precisely determined for those systems to be useful in QIP applications; e.g. for their use of spin-chirality qubits we need a precise determination of the size of the interdoublet energy separation, $\Delta$, but also of its nature, i.e. the determination of antisymmetric exchange interactions that would affect the chiral texture of the low-lying doublets.

Later on, it was shown that in addition to those two effects, a distribution of the effective Hamiltonian parameters was necessary to explain the broad features of the $g_{\perp}$ part of the spectra in $[3 \mathrm{Fe}-4 \mathrm{~S}]^{+}$clusters in ferredoxins; initial attempts considering strains of the zfs parameter of iron(III) yielded unreasonably high $D / J$ values ${ }^{26,27}$ (in light of the high $J$ values later determined) and it was then concluded that $J$-strains in combination with antisymmetric exchange could explain such broadenings with physically meaningful values of the effective Hamiltonian parameters. ${ }^{28}$ Subsequently, it was found that this attribute constitutes a recurring theme in several cases of $\mathrm{Cu}^{\mathrm{II}}{ }^{29,30} \mathrm{Fe}^{\mathrm{III}}{ }^{31-33}$ and $\mathrm{Cr}^{\mathrm{III}}{ }^{34,35}$ triangles, while distributions of electronic parameters are also beginning to emerge as a common theme in diverse inorganic materials, from simple paramagnetic complexes, ${ }^{36}$ to Metal-Organic Frameworks, ${ }^{37}$ Single-Molecule Magnets ${ }^{38-}$ ${ }^{40}$ and magnetic nanoparticles. ${ }^{41}$ These usually neglected effects complicate the situation and need to be explicitly addressed for a full description of the spin Hamiltonian of these complexes. While in all previous cases of antiferromagnetic triangles a phenomenological approach was used to describe the profile of the $g_{\perp}$ distributions, no attempt was made to correlate that profile to the values and distributions of more fundamental spin Hamiltonian parameters. 
Strain effects should, in principle, be rooted to structural effects within the cluster or the crystal, which ultimately influence superexchange through their effect on atomic orbital overlaps and transfer integrals. However, the precise mechanism is not always straightforward to extract and confirm, as several usually employed techniques, such as magnetic susceptometry and magnetic heat capacity can only reveal average values of the spin Hamiltonian parameters. Incoherent Inelastic Neutron Scattering (IINS) can reveal the precise positions of low-lying magnetic states, but due to its large intrinsic linewidths, it cannot discern closely spaced levels and narrow distributions of magnetic parameters. Mössbauer spectroscopy is a local technique that can distinguish between minute energy differences and which can reveal extremely weak interactions and narrow distributions, ${ }^{28,31}$ but it is limited to materials containing magnetic sites of elements with Mössbauer-active isotopes.

EPR spectroscopy is ideally suited for the study of magnetic asymmetries in spin triangles; since the ground state EPR signals are extremely sensitive to minute changes in $\Delta J=J-J^{\prime}$ and $|\mathbf{G}|$ (see below), not only can EPR measure small values of those parameters, but it can also reveal relatively narrow distributions of their central values. ${ }^{31}$

We have previously demonstrated the power of EPR spectroscopy in that respect, through the analysis of magnetic susceptibility and EPR data of $\left[\mathrm{Fe}_{3} \mathrm{O}(\mathrm{PhCOO})_{6}(\mathrm{py})_{3}\right]\left(\mathrm{ClO}_{4}\right) \cdot \mathrm{py}$, a triferric spin triangle with crystallographically-imposed $D_{3 h}$ symmetry. This complex exhibits magnetic asymmetries despite its perfectly equilateral molecular symmetry, as evidenced by synchrotron crystallography down to $4.5 \mathrm{~K}^{32}$ To analyse the distributions of the isotropic exchange couplings, $J_{i j}$, within this molecule we used a microscopically-derived model which takes into account the atomic vibration of the central oxide, considered to be the main superexchange pathway. ${ }^{42}$ Calculations of the atomic orbital overlaps allowed the derivation of expressions for 
the superexchange parameters and, subsequently, the calculation of the corresponding magnetic susceptibilities and EPR spectra. While in that study we considered the antisymmetric exchange vector as not subject to strain effects, such effects should also apply since it has been shown that it exhibits an angular dependence on the bridging $\mathrm{M}-\mathrm{X}-\mathrm{M}$ angle, where $\mathrm{X}$ is the bridging atom. ${ }^{43}$ To our knowledge, no studies have explicitly considered the effect of antisymmetric exchange distributions, either through microscopic or through phenomenological models.

The $\mathrm{Cr}^{\mathrm{III}}$ analogue of this complex, $\left[\mathrm{Cr}_{3} \mathrm{O}(\mathrm{PhCOO})_{6}(\mathrm{py})_{3}\right]\left(\mathrm{ClO}_{4}\right) \cdot 0.5 \mathrm{py}(\mathbf{1}$, Figure 1$)$, exhibits a similar crystallographically-imposed $D_{3 h}$ symmetry (Figure S1). The complex crystallises in the hexagonal $P 6_{3} / m$ space group, with two molecules per unit cell. Crystallographic 3-fold axes run through the central oxides of the $\left[\mathrm{Cr}_{3} \mathrm{O}(\mathrm{PhCOO})_{6}(\mathrm{py})_{3}\right]^{+}$cations, whereas the $\mathrm{Cr}_{3}$ planes coincide with crystallographic mirror planes. The coincidence of the atoms with these special positions imposes a molecular $D_{3 h}$ point-group symmetry to the cations, essentially transmitting the crystallographic symmetry to the molecular level. With respect to the local site symmetry (LSS), the Cr atoms adopt an almost axially distorted octahedral coordination sphere, with a compressed Cr- $\mathrm{O}_{\text {ox }}$ bond (1.913 $\AA$ ) and an elongated $\mathrm{Cr}-\mathrm{N}_{\text {py }}$ bond, and with the $\mathrm{Cr}$ atom $0.178 \AA$ below the equatorial plane defined by the four carboxylate oxygen atoms. The LSS can be considered as quasi- $C_{2 v}$ disregarding the small trigonal distortion inferred by the slight non-equivalence of atoms $\mathrm{O}(2)$ and $\mathrm{O}(3)$.

This crystallographically imposed molecular symmetry renders this complex very attractive for such a study: eventual magnetic asymmetries are bound to be intrinsic, i.e. present at the highest possible symmetries, and not due to structural deformations related to a lowering of the molecular symmetry by the ligands, counterions and solvate molecules that lead to crystallographically inequivalent metal sites. It is therefore an equally interesting system for the 
study of such effects. Moreover, the previous IINS studies on this complex, ${ }^{44}$ which have revealed finer details on the magnetic structure of this complex, are invaluable for crossverification of the conclusions of EPR data. In this work, we develop a methodology for deriving detailed profiles of the spin Hamiltonian parameters of spin triangles from EPR data, to a level of detail unattainable by other techniques, which extends to the description of their specific distributions (strains).

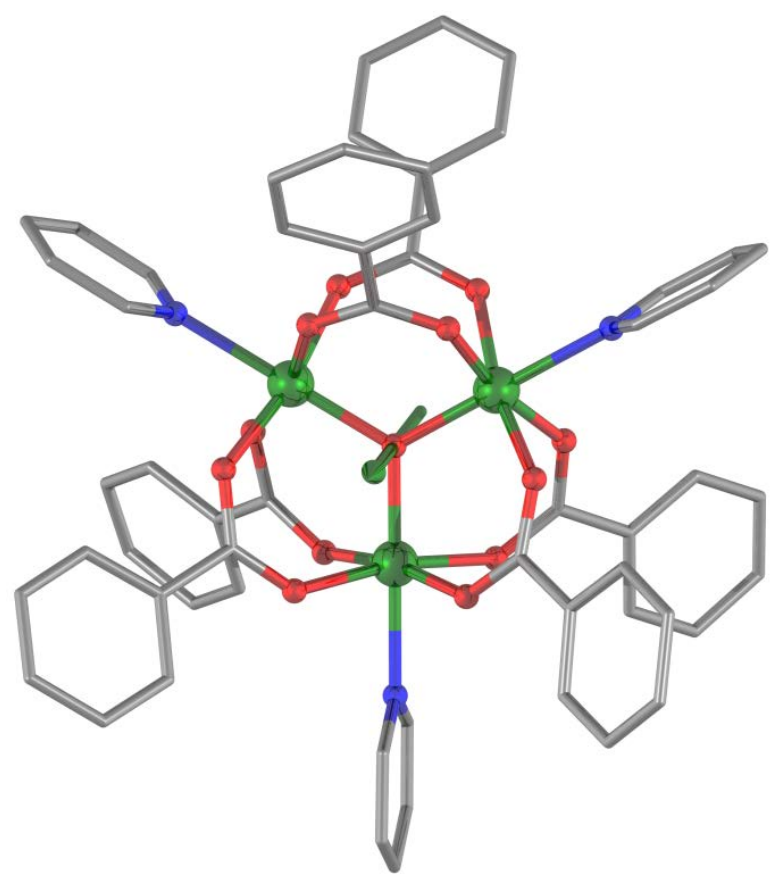

Figure 1. POV-ray plot of the cation of complex 1. The green arrow indicates the crystallographically-imposed $C_{3}$ symmetry axis, which is parallel to the crystallographic $c$-axis. 


\section{Experimental}

\section{Synthesis}

All reagents and solvents were used as received. Caution: Though no such tendency was observed during the current work, perchloric acid and perchlorate salts are potentially explosive and should be handled in small quantities and not be heated to dryness.

Complex 1 had been previously synthesized by reaction of chromium(III) perchlorate with a pyridine solution of benzoic acid. ${ }^{44}$ In the current work, we prepared this complex from a $\mathrm{Cr}_{3}^{\mathrm{III}}$ precursor formed by reaction of a solution of $\mathrm{CrCl}_{3} \cdot 6 \mathrm{H}_{2} \mathrm{O}(20.0 \mathrm{mmol}, 5.33 \mathrm{~g})$ in $\mathrm{H}_{2} \mathrm{O}(20 \mathrm{~mL})$, and a solution of $\mathrm{PhCOOH}(44.0 \mathrm{mmol} 5.37 \mathrm{~g})$ and $\mathrm{NaHCO}_{3}(44.0 \mathrm{mmol}, 3.70 \mathrm{~g})$ in $\mathrm{H}_{2} \mathrm{O}$ (80 $\mathrm{mL}$ ). The blue-gray precipitate that formed almost instantly was filtered through a glass frit, washed with $\mathrm{H}_{2} \mathrm{O}$ until the filtrates were colorless, and dried at $65^{\circ} \mathrm{C}$ overnight to yield a powder formulated as $\left[\mathrm{Cr}_{3} \mathrm{O}\left(\mathrm{O}_{2} \mathrm{CPh}\right)_{6}\left(\mathrm{H}_{2} \mathrm{O}\right)_{3}\right]\left(\mathrm{PhCO}_{2}\right) \quad(5.1 \mathrm{~g}, 71 \%$ yield $)$. While no detailed characterization of this starting material was pursued, its X-band EPR spectrum at $4.2 \mathrm{~K}$ exhibited the characteristics of trinuclear antiferromagnetically coupled complexes (Figure S2). $2.00 \mathrm{~g}$ (1.86 mmol) of this powder were dissolved in py $(15 \mathrm{~mL})$ to yield a dark blue solution, to which were added $1.44 \mathrm{~mL}(16.74 \mathrm{mmol})$ of $70 \% \mathrm{HClO}_{4}$ in small aliquots to avoid excessive heating. Occasional fizzling, with the formation of a white crystalline powder considered to be (pyH) $\left(\mathrm{ClO}_{4}\right)$, was observed on the flask walls upon additions. The solution was then refluxed for $3.5 \mathrm{~h}$, over the course of which it became purple and precipitated a green-sage powder. The powder was filtered on a glass frit, washed with $\mathrm{Et}_{2} \mathrm{O}$ until the smell of pyridine disappeared and dried under vacuum for 10 min. Microanalytical and powder XRD (Figure S3) data confirmed this as complex 1. Elemental analysis for $\mathrm{C}_{59.5} \mathrm{H}_{47.5} \mathrm{Cr}_{3} \mathrm{~N}_{3.5} \mathrm{O}_{17} \mathrm{Cl}$ : Calc. C, 56.05; H, 3.76; N, 3.85. Found C, 55.34; H, 3.72; N, 4.51. 


\section{Physical measurements}

Elemental analyses (C, H, N) were performed at the Service d'analyses, de mesures physiques et de spectroscopie optique of the University of Strasbourg. Powder X-ray diffraction diagrams were collected at $293 \mathrm{~K}$ on a Bruker D8 diffractometer using monochromatic $\mathrm{Cu}-\mathrm{K} \alpha$ radiation with a scanning range between 4 and $50^{\circ}$ using a scan step of $2 \% \mathrm{mn}$. The simulated diagram is based on single-crystal data collected at $173 \mathrm{~K}$. SQUID data were collected on a Quantum Design MPMS3 magnetometer. Isothermal variable-field magnetization measurements confirmed the absence of ferromagnetic impurities. Data were corrected for the sample holder and TIP contributions, and diamagnetism was estimated by using Pascal constants. The magnetic susceptibilities were computed by exact calculation of the energy levels associated with the spin Hamiltonian through diagonalisation of the full matrix using Phi 3.1.1. ${ }^{45}$ Residuals, $R$, for the fits are the ones defined in the Phi documentation. The paramagnetic impurity contribution was calculated assuming a $S=3 / 2$ species fraction $\rho$, so that $\chi_{M}{ }^{\text {total }}=(1-\rho) \chi_{M}{ }^{\mathrm{Cr} 3}+\rho \chi_{M}{ }^{\mathrm{par}}$. X-band (9.311 GHz) EPR spectra were collected on an EMXplus spectrometer fitted with an EMX microX bridge and a Bruker ER4122SHQE cavity operating in the $\mathrm{TE}_{011}$ mode. For low temperature experiments, the cavity was fitted with an ESR900 dynamic continuous flow cryostat controlled with an Oxford ITC503S Intelligent Temperature Controller. To confirm that the broadenings are intrinsic to the material and independent of crystallite size, orientation and other extraneous effects, spectra were collected from the pristine "as-made” powder and from a powder having been carefully ground in a mortar. The powders were then tightly packed in the EPR tube with cotton to prevent torqueing and spectra were collected under different rotations of the EPR sample tube. Pristine and ground powders gave identical spectra at all rotations. Spectra were fitted with Easyspin v5.2 $2^{46}$ using custom-made routines. 


\section{Results}

\section{Magnetic susceptibility studies}

The $\chi_{M} T$ product at $300 \mathrm{~K}$ is $4.35 \mathrm{~cm}^{3} \mathrm{~mol}^{-1} \mathrm{~K}$, significantly below the value of three noninteracting $S=3 / 2$ spins $\left(5.51 \mathrm{~cm}^{3} \mathrm{~mol}^{-1} \mathrm{~K}, g=1.98\right)$, indicating the interplay of antiferromagnetic interactions. This is corroborated by the decrease of $\chi_{M} T$ upon cooling, down to a plateau of $0.41 \mathrm{~cm}^{3} \mathrm{~mol}^{-1} \mathrm{~K}$ at $1.8 \mathrm{~K}$, slightly above the value of a $S=1 / 2$ system $\left(0.37 \mathrm{~cm}^{3}\right.$ $\mathrm{mol}^{-1} \mathrm{~K}, g=1.98$ ). This comparatively large value can be attributed to the simultaneous presence of a small fraction of paramagnetic impurity. This is in line with the fact that the magnetization at 1.8 approaches saturation $(7 \mathrm{~T})$, at a value of $1.03 N_{A} \mu_{B}$, slightly above the saturation value of a $S_{\mathrm{T}}=1 / 2$, i.e. the ground state predicted for an antiferromagnetically coupled triangle of halfinteger spins (see below). EPR spectroscopic data agree with the presence of a small fraction of a $S=3 / 2$ paramagnetic impurity (see below).

The spin Hamiltonian we employed, considers an isosceles magnetic symmetry and antisymmetric exchange interactions, as expressed by Equation 1:

$$
\hat{H}=-2 J\left(\hat{\mathbf{S}}_{1} \hat{\mathbf{S}}_{2}+\hat{\mathbf{S}}_{2} \hat{\mathbf{S}}_{3}\right)-2 J^{\prime} \hat{\mathbf{S}}_{1} \hat{\mathbf{S}}_{3}-2 \mathbf{G}\left(\hat{\mathbf{S}}_{1} \times \hat{\mathbf{S}}_{2}+\hat{\mathbf{S}}_{2} \times \hat{\mathbf{S}}_{3}+\hat{\mathbf{S}}_{3} \times \hat{\mathbf{S}}_{1}\right)+\mu_{B} g \mathbf{H} \sum_{i=1}^{3} \hat{\mathbf{S}}_{i}
$$

Moreover, based on symmetry considerations for this type of molecules, we consider that $G_{z}$ $>>\left(G_{x}, G_{y}\right) \approx 0$, which means that $|\mathbf{G}|=G_{z}$. Single-ion zero-field splitting terms for the $\mathrm{Cr}^{\mathrm{III}}$ ions

were considered, but in view of the high quality of the fits based on the exchange-only Hamiltonian, and the negligible effect of such terms on the EPR spectra (see below), such terms were not introduced.

Fits were simultaneously carried out on the $\chi_{M} T$ vs $T$ and the $M$ vs $H$ data (Table 1 ) and the best fit is shown in Figure 2. Initial fits assuming a simple equilateral model $(J=J)$ and a 
fraction $\rho$ of a $S=3 / 2$ paramagnetic impurity of molecular weight equal to that of $\mathbf{1}$, yielded best-fit solution $J=-10.8 \mathrm{~cm}^{-1}, G_{z} \sim 0, g=2.0, \rho=1.6 \%$, indicating that the first $S_{\mathrm{T}}=3 / 2$ state will lie $3 J_{\mathrm{av}}=32.4 \mathrm{~cm}^{-1}$, in excellent agreement with the value of $32.6 \mathrm{~cm}^{-1}$ previously determined for this complex by IINS experiments with neutrons of $2.35 \AA$ wavelength. ${ }^{44}$ Fits without the inclusion of the impurity yielded inferior quality fits and abnormally high $g$ values.

Table 1. Best-fit solutions to the magnetic susceptibility data of $\mathbf{1}$, assuming an equilateral or the two types of isosceles magnetic symmetries. For each symmetry, two solutions are considered, one without antisymmetric exchange interactions $\left(G_{z}=0\right.$, fixed), and one with $G_{z} \neq 0$ (free variable). For the $J, J^{\prime}$ and $J_{\text {av }}$ parameters, significant digits beyond the third are below the precision of the technique, but only presented (italics) to show the proximity of best-fit values.

\begin{tabular}{llllllll}
\hline & $J\left(\mathrm{~cm}^{-1}\right)$ & $J^{\prime}\left(\mathrm{cm}^{-1}\right)$ & $J_{a v}\left(\mathrm{~cm}^{-1}\right)$ & $G_{z}\left(\mathrm{~cm}^{-1}\right)$ & $\rho$ & $g$ & $R$ \\
\hline$J=J^{\prime}$ & -10.8 & - & -10.8 & $\mathbf{0}$ (fixed) & $1.6 \%$ & 2.01 & $8.1 \times 10^{-7}$ \\
& -10.8 & - & -10.8 & 0.0095 & $1.6 \%$ & 2.01 & $2.9 \times 10^{-7}$ \\
$|J|>\left|J^{\prime}\right|$ & -10.83 & -10.82 & -10.83 & $\mathbf{0}$ (fixed) & $1.6 \%$ & 2.01 & $5.0 \times 10^{-7}$ \\
& -10.88 & -10.84 & -10.86 & 0.051 & $1.8 \%$ & 2.01 & $2.9 \times 10^{-8}$ \\
$|J|<\left|J^{\prime}\right|$ & -10.82 & -10.83 & -10.83 & $\mathbf{0}$ (fixed) & $1.6 \%$ & 2.01 & $3.1 \times 10^{-7}$ \\
& -10.828 & -10.832 & -10.83 & 0.011 & $1.6 \%$ & 2.01 & $2.8 \times 10^{-7}$ \\
\hline
\end{tabular}

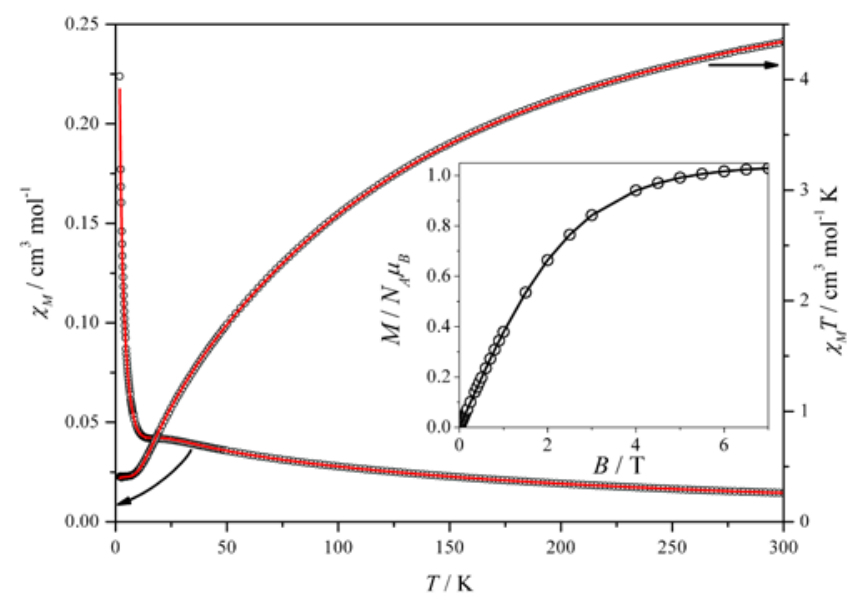


Figure 2. Magnetic susceptometry data (open circles) for complex 1: $\chi_{M} T$ vs $T$ data at $5 \mathrm{kG}$ and $M$ vs $H$ data at $2 \mathrm{~K}$ (inset) for complex 1 . Lines show the best fit according to the model described in the text.

The very high quality of the fits meant that lower-symmetry models $\left(J \neq J^{\prime}\right)$ were likely to become overparametrised. Indeed, by liberating this constrain we only obtained a marginal improvement, while the $J$ parameters coalesced to values practically equal to the previously determined $J_{\mathrm{av}}$. Simulations considering various isosceles conformations with $\Delta J$ ranging from -0.6 to $+0.6 \mathrm{~cm}^{-1}$ produced practically superimposable $\chi_{M} T$ vs $T$ and the $M$ vs $H$ curves (Figure S5), which indicates that the magnetic susceptibility of this complex mostly depends on $J_{\text {av }}$ and less on the individual $J$ and $J^{\prime}$ values. Introduction of the antisymmetric exchange component $G_{z}$ to the Hamiltonian improved the fits, yielding very small $G_{z}$ values $\left(\sim 0.01-0.05 \mathrm{~cm}^{-1}\right)$. The bestfit solution assuming an isosceles model and antisymmetric exchange was $J=-10.88 \mathrm{~cm}^{-1}, J^{\prime}=$ $-10.84 \mathrm{~cm}^{-1} G_{z}=0.051, g=2.0, \rho=1.8 \%$,

To assess the sensitivity of the fits to each parameter, error plots were constructed based on this solution, by varying $J$ or $G_{z}$ and keeping all other parameters fixed. These indicated that while the error curve exhibited a relatively sharp minimum for $G_{z}$ variations, the corresponding minimum was less well defined for $J$ (Figure S6). These observations lead us to the conclusion that magnetic susceptometry is an excellent tool for the determination of the $J_{\text {av }}$ value in this system, and reasonably good at determining $G_{z}$. However, it is ineffective in the determination of magnetic asymmetries $(\Delta J)$.

In comparing 1 to its ferric analogue, ${ }^{47}$ we observe that, with the exception of the $\mathrm{M}-\mathrm{O}_{\text {охо }}$ bond, the coordination sphere is significantly smaller, as evidenced by the shorter M-L bond lengths (Table 2) and that the magnetic exchange coupling is half in strength $\left(J_{\mathrm{av}}^{\mathrm{Fe}}=-21.5 \mathrm{~cm}^{-1}\right)$. This 
latter attribute can be explained by the differences in electronic configurations of $\mathrm{Cr}^{\mathrm{III}}$ and $\mathrm{Fe}^{\mathrm{III}}$ octahedrally coordinated ions. $\mathrm{Cr}^{\mathrm{III}}$ is a $\mathrm{d}^{3}$ ion which, in quasi-octahedral environments, adopts a $\left(t_{2 g}\right)^{3}\left(e_{g}\right)^{0}$ electronic configuration, with empty $\mathrm{d}_{z^{2}}$ and $\mathrm{d}_{x^{2}-y^{2}}$ orbitals, while $\mathrm{Fe}^{\mathrm{III}}$ is a $\mathrm{d}^{5}$ ion with a $\left(t_{2 g}\right)^{3}\left(e_{g}\right)^{2}$ electronic configuration, with those orbitals half-filled. Since the $\mathrm{d}_{z^{2}}$ orbital in this topology is the most effective in transmitting superexchange between metal ions, with $d_{x z}$ and $d_{y z}$ also active but less so, the interactions between ferric ions are stronger. ${ }^{42}$

Table 2. Comparison of metal-ligand interatomic distances (in $\AA$ ) in $\mathbf{1}$ and its ferric analogue.

\begin{tabular}{|c|c|c|c|}
\hline & $\begin{array}{l}M^{I I I}=C r^{I I I}(1) \\
\left(173 \text { K, ref. }{ }^{44}\right)\end{array}$ & $\begin{array}{c}\mathbf{M}^{\mathrm{III}}=\mathrm{Fe}^{\mathrm{III}} \\
\left(233 \mathrm{~K}, \text { ref. }^{47}\right)\end{array}$ & $\begin{array}{c}M^{I I I}=F^{I I I I} \\
\left(90 \mathrm{~K} \text {, ref. }^{32}\right)\end{array}$ \\
\hline $\mathrm{M}(1)-\mathrm{O}(1)$ & 1.913 & $1.9149(10)$ & $1.9080(2)$ \\
\hline $\mathrm{M}(1)-(\mathrm{N} 1)$ & 2.144 & $2.206(6)$ & $2.1946(12)$ \\
\hline $\mathrm{M}(1)-\mathrm{O}(2)$ & 1.972 & 2.014(3) & $2.0107(7)$ \\
\hline $\mathrm{M}(1)-\mathrm{O}(3)$ & 1.968 & 2.015(3) & 2.0072(8) \\
\hline
\end{tabular}

\section{X-band EPR spectroscopy studies}

While magnetic susceptometry is unsuited for the determination of $\Delta J$, and only partially effective to discern small values of $G_{z}$, X-band EPR spectroscopy is ideally suited for this task. Even very weak antisymmetric exchange interactions, can induce strong anisotropy when combined with magnetic asymmetries $(\Delta J)$. In such a situation, the perpendicular component becomes shifted to higher fields $(g<<2)$; in fact, the smaller the asymmetry, the stronger will be the effect of AE. 
The origin and position of these signals have been previously analyzed in detail; ${ }^{24}$ assuming the $-2 J_{i j} S_{i} S_{j}-2 \mathbf{G}\left(S_{i} \times S_{j}\right)$ formalism and for $S_{i}=3 / 2$ spins, these are given by the expressions 2 and 3:

$$
\begin{aligned}
& g_{\| \text {eff }}=g_{\| 0} \\
& g_{\perp \text { eff }}=g_{\perp 0} \sqrt{\frac{16|\Delta J|^{2}-(h v)^{2}}{\Delta^{2}-(h v)^{2}}}
\end{aligned}
$$

where $g_{\| \text {eff }}\left(g_{\perp e f f}\right)$ are the experimentally observed $g_{\|}\left(g_{\perp}\right)$ values, $g_{\| 0}\left(g_{\perp 0}\right)$ are the intrinsic singleion $g_{\|}\left(g_{\perp}\right)$ values, $h v$ is the microwave energy, $\Delta J=J-J, \Delta=\left[16(\Delta J)^{2}+|\mathbf{D}|^{2}\right]^{1 / 2}$, and $\mathbf{D}=$ 8G $\sqrt{ } 3$. The $\Delta J$ parameter indicates the magnetic asymmetry due to Heisenberg isotropic interactions and $\Delta$ is the energy separation between the low-lying doublets stemming both from the magnetic asymmetry and antisymmetric exchange; in effect, if $\Delta J=0$ then $\Delta=|\mathbf{D}|$ and if $|\mathbf{D}|$ $=0$ then $\Delta=\Delta J$. These expressions reveal that the $g_{\perp \text { eff }}$ positions are extremely sensitive to even small variations of $\Delta J$ and $\Delta$, and in extension to those of $G_{z}$.

We note here that we also considered the effect of single-ion zfs of the $\mathrm{Cr}^{\mathrm{III}}$ ions on the EPR spectrum of $\mathbf{1}$, and their possible utility in reproducing the broadenings of the $g_{\perp}$ resonance. $\mathrm{Cr}^{\mathrm{III}}$ ions are reported to exhibit single-ion zfs between +0.2 and $+1 \mathrm{~cm}^{-1}{ }^{48}$ To test the sensitivity of the $g_{\perp}$ resonances of $\mathbf{1}$ to $D$-strains we simulated the EPR spectra of $\mathbf{1}$ assuming the central values determined for its exchange parameters (see below), i.e. $J=-10.7 \mathrm{~cm}^{-1}, J^{\prime}=-11.0 \mathrm{~cm}^{-1}$ (i.e. $J_{a v}-10.8 \mathrm{~cm}^{-1}$ ) and $G_{z}=0.041 \mathrm{~cm}^{-1}$; the line widths were those determined below. We then varied the value of $D$, whose $z$-axis is assumed to point toward the center of the triangle. We considered a value in the middle of the reported range, i.e. $+0.6 \mathrm{~cm}^{-1}$, as representative of a probable situation. Simulations indicated a shift of $23 \mathrm{G}$ for a change in $D$ from 0 to $+0.6 \mathrm{~cm}^{-1}$, whereas the $g_{\perp}$ resonance of $\mathbf{1}$ spans a magnetic field range of almost 2000 G (Figure S7). 
Clearly, $D$-strains of any realistic width cannot account for such a broad resonance. This observation validates our choice of not considering $\mathrm{zfs}$ of $\mathrm{Cr}^{\mathrm{III}}$ ions in the simulation of EPR spectra.

The X-band EPR spectrum at $4.3 \mathrm{~K}$ (Figure 3) reveals an axial signal consisting of a sharp peak at $g=1.97$, attributed to the $g_{\|}$component of the $S_{\mathrm{T}}=1 / 2$ ground state, and a broad derivative feature spanning the 1.97 to $\sim 1.2 g$-range, with the trough minimum at $g=1.77$; this is attributed to the $g_{\perp}$ component of the ground state signal.

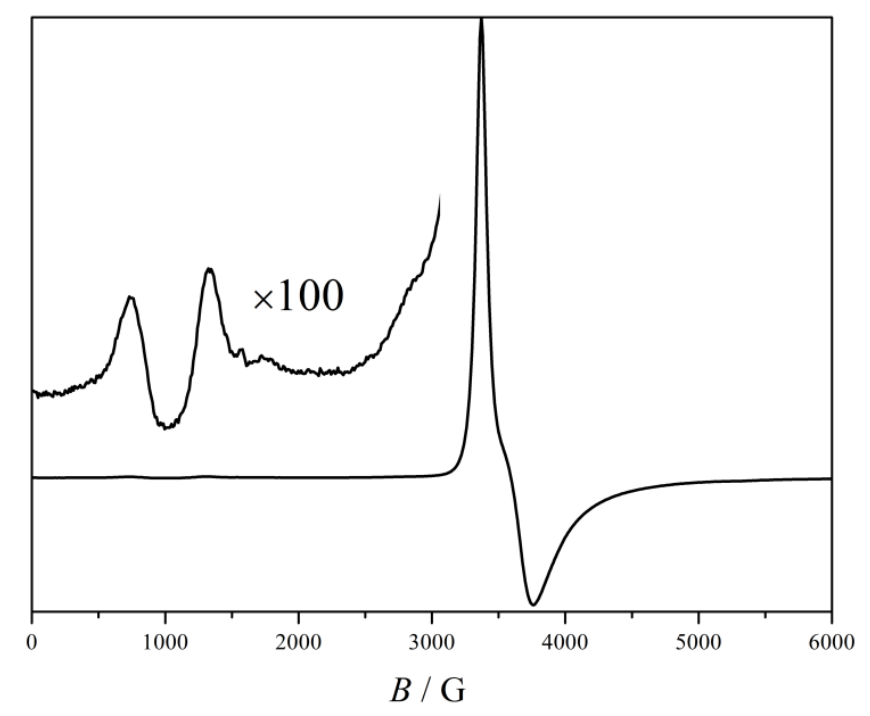

Figure 3. X-band EPR spectrum of $\mathbf{1}$ at $4.3 \mathrm{~K}$. The inset shows the weak resonances assigned to a small fraction of paramagnetic impurity. Experimental conditions: $f_{\mathrm{EPR}}=9.311 \mathrm{GHz}$, mod. ampl. $=10 \mathrm{G}_{\mathrm{pp}}$, microwave power $=0.0196 \mathrm{~mW}$.

Weak signals at low fields are attributed to a small percentage of $S=3 / 2$ paramagnetic impurity. Two other possibilities for these resonances were also considered, but discarded (see Scheme 1). The first is that these signals arise from the excited $S_{\mathrm{T}}=3 / 2$ state. However, this is too high in energy $\left(\sim 32 \mathrm{~cm}^{-1}\right)$ for a significant thermal population at the experimental temperature $\left(\sim 1.5 \times 10^{-10}\right.$ relative population at $\left.4.3 \mathrm{~K}\right)$. Such signals are observed at higher 
temperatures ( $\sim \mathrm{K})$, but at different regions and exhibiting a different $I \times T$ thermal dependence (Figure S4). The other possibility is that these signals correspond to interdoublet transitions, between the Zeeman sublevels of the low-lying spin doublets (i.e. $\left|S_{12}=0, M_{S}= \pm 1 / 2\right\rangle$ and $\mid S_{12}=$ $\left.1, M_{S}=\mp 1 / 2\right\rangle$, where $S_{12}$ is the quantum number of the intermediate spin operator $\mathbf{S}_{12}=\mathbf{S}_{1}+\mathbf{S}_{2}$ ). However, the required spacing for such resonances to occur at the X-band would be extremely small, contradicting the conclusions of IINS studies with neutrons of $6.5 \AA$ wavelength, which have convincingly demonstrated a range of interdoublet separations $1.13 \mathrm{~cm}^{-1}<\Delta<2.6 \mathrm{~cm}^{-1}{ }^{44}$ We are therefore confident that these signals arise from a small fraction of a $S=3 / 2$ paramagnetic impurity.

Scheme 1. Zeeman diagrams showing characteristic parameters and resonances $\left(v_{\mathrm{EPR}}=9.3 \mathrm{GHz}\right.$, $h v_{\mathrm{EPR}}=0.31 \mathrm{~cm}^{-1}$ ) in two indicative cases for $\mathbf{B}_{0} \| z$ (black) and $\mathbf{B}_{0} \perp z$ (red). Left: For for $J=-10.8$ $\mathrm{cm}^{-1}, J^{\prime}=-11.2 \mathrm{~cm}^{-1}\left(\Delta J=0.4 \mathrm{~cm}^{-1}, J_{\mathrm{av}}=-10.93 \mathrm{~cm}^{-1}\right)$ and $G_{z}=0.04 \mathrm{~cm}^{-1}$ the interdoublet gap is $\Delta=1.7 \mathrm{~cm}^{-1}$ and only the intradoublet transitions occur. Right: for $J=-10.9 \mathrm{~cm}^{-1}, J^{\prime}=-11 \mathrm{~cm}^{-1}$ $\left(\Delta J=0.1 \mathrm{~cm}^{-1}, J_{\mathrm{av}}=-10.93 \mathrm{~cm}^{-1}\right)$ and $G_{z}=0.04 \mathrm{~cm}^{-1}$, the interdoublet gap is $\Delta=0.6 \mathrm{~cm}^{-1}$ and additional interdoublet transitions arise (dotted lines). The thickness of the lines indicates the intensity of the EPR transitions based on thermal occupancies. Transitions within the excited quartet are of negligible intensity at $4 \mathrm{~K}$. The quartet zfs is a second-order effect due to antisymmetric exchange and is observed in triangles of $S_{i}>1 / 2$. For simplicity, at $\mathbf{B}_{0} \perp z$ the first quartet is omitted for the large $\Delta$ model due to the presence of multiple anticrossings with other closely spaced quartet states. 

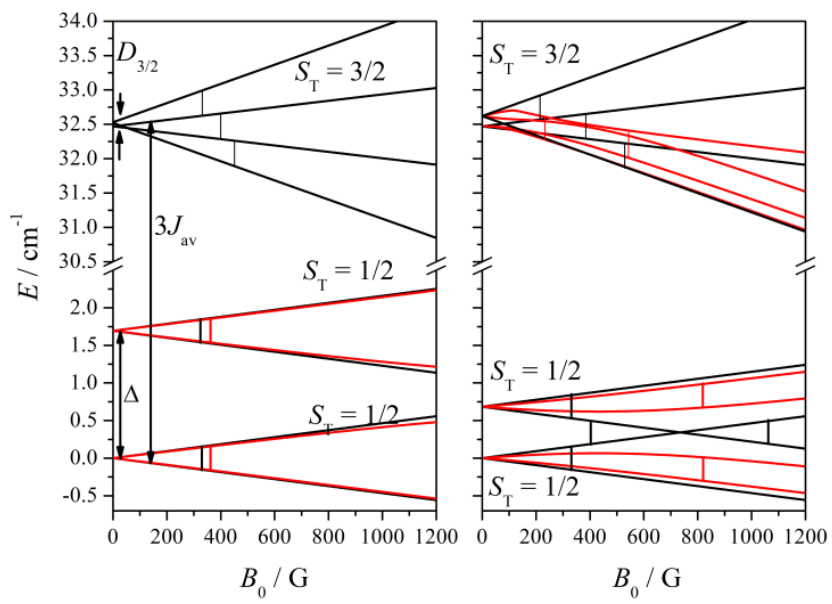

\section{Implementation of the dynamic model}

For a complete description of the multispin Hamiltonian, we need to assess the distributions of the effective parameters that influence the $g_{\perp \text { eff }}$ positions, i.e. the $J_{i j}$ parameters and their differences, and $G_{z i j}$. One method is to start from microscopic considerations in order to construct a model for the distributions of such parameters from first principles. Based on that approach, we had previously analyzed the EPR and magnetic susceptibility data of the ferric analogue of this complex through a dynamic spin Hamiltonian, which takes into account the thermal vibrations of the central oxide. This oxide provides the main superexchange pathway between the triangle's spins. By employing previously developed magnetostructural correlations, ${ }^{42}$ we modelised the effect of atomic vibrations on the $J_{i j}$ couplings and we were able to reproduce the features of the $g_{\perp}$ signal. $^{32}$ Based on the observation of several discrete $g_{\perp}$ resonances, we made the assumption of preferential orientations of the oxide deformation vector. Indeed, by assuming preferential orientations of the oxide deformation vector at every $30^{\circ}$, we were able to reproduce those spectral features; consideration of additional subspectra, i.e. no preferential orientations, led to a double-trough spectrum, with the two troughs 
corresponding to the two isosceles magnetic conformations $(|J|>|J|,|J|<|J|)$, due to the averaging-out of intermediate angles corresponding to scalene magnetic conformations.

In the present case, the $g_{\perp}$ signal has a very different appearance from that of the ferric analogue: it is constrained to a higher $g$ range (lower magnetic fields), and is much smoother and featureless. Use of the previously mentioned dynamic model ${ }^{32}$ could not reproduce its spectral features, as it led to very narrowly distributed $g_{\perp}$ signals (Figure S8). A discernible separation of the $g_{\perp}$ signals corresponding to the two isosceles conformations $(|J|<|J|$ and $|J|>|J|)$ could only be achieved for high $G_{z}$ values $\left(\sim 0.8 \mathrm{~cm}^{-1}\right)$, which required large radial deformation parameters of the central oxide ( $a=0.22$; the parameter is defined in ref. 32), corresponding to abnormally

high $\Delta J$ values $\left(4.5 \mathrm{~cm}^{-1}\right)$. Even under these conditions, when considering a large number of subspectra, the calculated spectra assumed a double-trough form, clearly inconsistent with the experimental one.

\section{Empirical determination of the $g_{\perp}$ distribution}

Following these attempts, and in the absence of another microscopically derived model, our next choice was to employ the reverse strategy, whereby we modelise the distributions of the respective parameters phenomenologically, and attempt to derive a microscopic physical meaning. An empirical analysis of the broadening of the $g_{\perp e f f}$ component was first presented by Sanakis et al. ${ }^{28}$ and subsequently applied in several other cases ${ }^{29-35}$ where significant antisymmetric exchange was present. In those studies, such signals were analysed through the consideration of a series of fictitious axial $S=1 / 2$ systems, of identical $g_{\|}$and distributed $g_{\perp}$ values.

We undertook a similar preliminary analysis and fitted our spectrum to a series of subspectra with a common $g_{\| e f f}=1.97$ and a distribution of $g_{\perp e f f}$ values (Figure 4). For the intrinsic linewidth 
of each sub-spectrum we were based on the lineshape of the $g_{\|}$feature, which is not broadened by exchange effects; best results were obtained with a Voigtian curve $\left(\sigma w_{\text {Gauss }}=5.7 \mathrm{mT}, \sigma w_{\text {Lor }}=\right.$ $2.3 \mathrm{mT})$. An additional $g$-strain on the perpendicular components $\left(\sigma g_{\perp}=0.02\right)$ was required to obtain a smooth line shape with a limited amount of spectra. The fitted distribution reveals a maximum density at $g_{\perp}=1.78$, consistent with the trough position of the experimental spectrum.

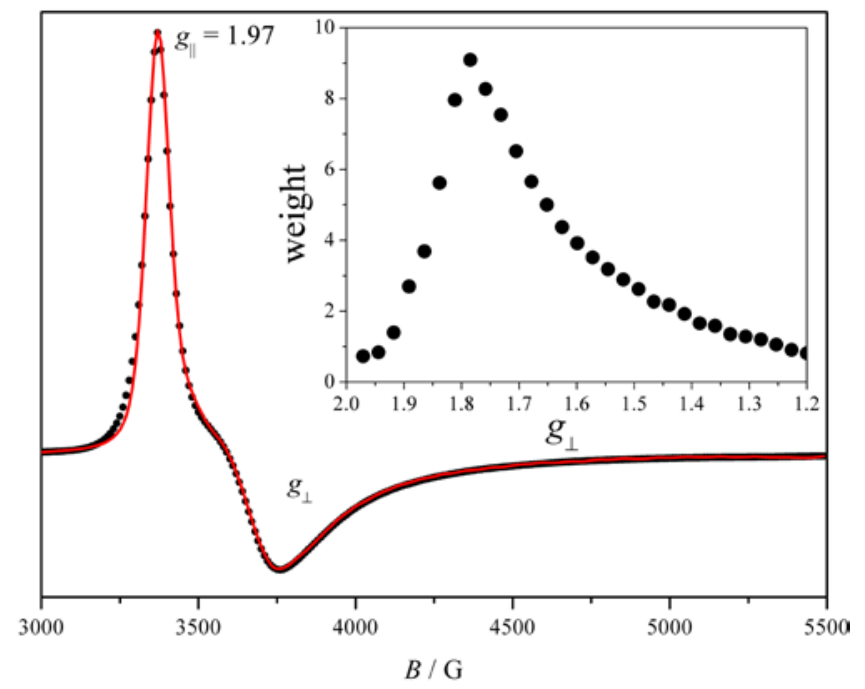

Figure 4. X-band EPR spectrum of $\mathbf{1}$ at $4.2 \mathrm{~K}$ (black points) and simulation (red line) based on a series of subspectra exhibiting a distribution of $g_{\perp}$ values (inset). Experimental conditions: $f_{\mathrm{EPR}}=$ 9.311 GHz, mod. ampl. $=10 \mathrm{Gp}$, microwave power $=0.0196 \mathrm{~mW}$.

\section{Empirical distributions of $\Delta J$ and $G_{z}$}

Based on the previously determined $g_{\perp e f f}$ distribution, we can derive effective distributions of $\Delta J$ (by assuming a given $G_{z}$ ) or effective distributions of $G_{z}$ (by assuming a given $\Delta J$ ). Indeed based on Equation 3 we can solve for either quantity with simple algebraic manipulations. Solving for $|\mathbf{G}|$ yields Equation 4 and solving for $\Delta J$ yields Equation 5. These can be used to derive each quantity from the $g_{\perp \text { eff }}$ value derived from an experimental EPR spectrum and by making a hypothesis of the other quantity: 
$|\mathbf{G}|=\frac{\sqrt{\left[16 \Delta J^{2}-(h v)^{2}\right]\left(1-\kappa^{2}\right)}}{8 \sqrt{3} \kappa}$

$\Delta J=\frac{1}{4} \sqrt{(h v)^{2}+\frac{\kappa^{2} 192|\mathbf{G}|^{2}}{1-\kappa^{2}}}$

where $\kappa=g_{\perp e f f} / g_{\perp 0}$.

Making a justified hypothesis of the central values based on IINS data (see Supporting Information) we can recalculate the derived distribution of $g_{\perp e f f}$ to a distribution of $\Delta J$ (assuming a unique $G_{z}$ ) or to a distribution of $G_{z}$ (assuming a unique $\Delta J$ ). These extreme cases consider that one parameter is subject to distribution, while the other is uniquely determined (Figure 5).
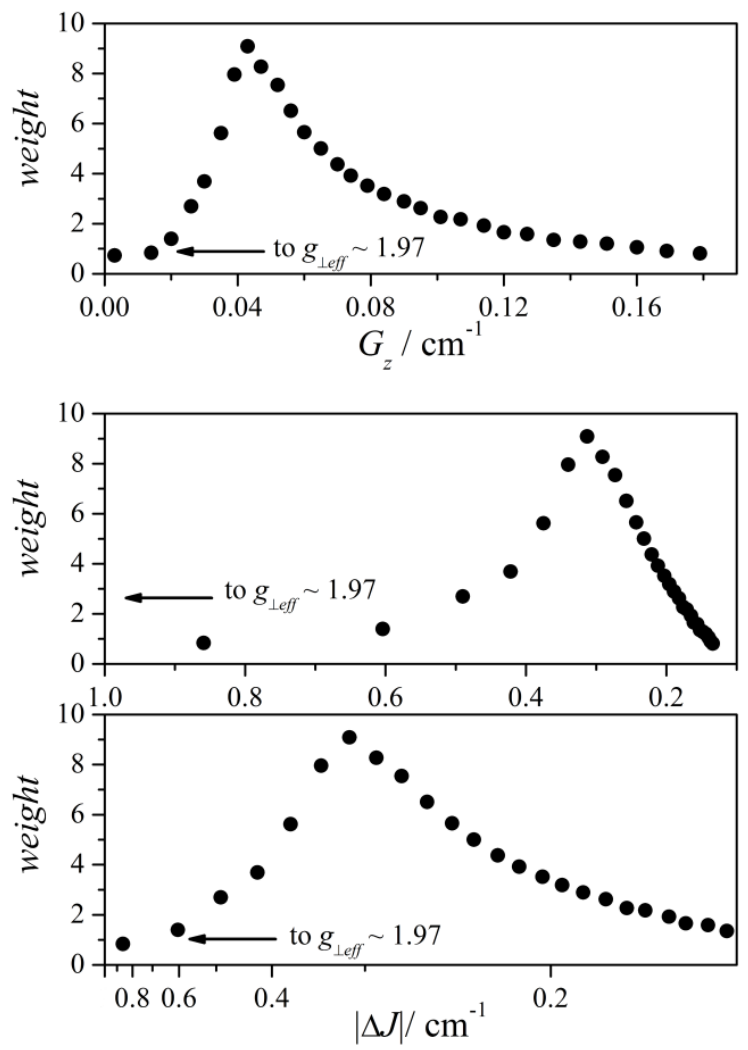

Figure 5. Distribution of $g_{\perp e f f}$ expressed as: (i) a distribution of $G_{z}$ for $\Delta J=0.3 \mathrm{~cm}^{-1}$ (top) and (ii) a distribution of $\Delta J$ for $G_{z}=0.041 \mathrm{~cm}^{-1}$ (middle and bottom). Due to the quasi-reciprocal relation between $g_{\perp e f f}$ and $\Delta J$ in the eqn, the evenly spaced domain in $g_{\perp e f f}$ space corresponds to a 
reciprocally spaced domain in $\Delta J$ space, with denser population of smaller $\Delta J$ values. For an illustration of the effect, the $\Delta J$ distribution is plotted on linearly (middle) and reciprocally (bottom) scaled axes. The arrows indicate the direction towards which the $g_{\perp \text { eff }}$ resonance tends to $g_{\perp 0}$ (isotropic spectra).

These representations of the empirically derived $g_{\perp \text { eff }}$ distribution provide us with a qualitative starting point for defining simultaneous distributions of the individual parameters indicating asymmetric distributions with narrow fronts and long tails, and a quasi-reciprocal spacing for $\Delta J$. Regarding the sign of the parameters, those simulations assume $\Delta J>0$, i.e. $|J|<|J|$. Simulations assuming $\Delta J<0$, i.e. $|J|>|J|$, are equally valid, but were not pursued in detail, as preliminary tests showed that the calculated spectra were practically identical to those with $\Delta J>$ 0 . This conclusion is in line with the conclusions derived by application of the dynamic model (see above), which indicated an indiscernible separation of the $g_{\perp}$ signals of the two isosceles conformations $(|J|<|J|$ and $|J|>|J|)$. As for the antisymmetric exchange vector, it was assumed that $G_{z}>0$, in accordance to theoretical calculations for exchange coupled $\mathrm{d}^{3}-\mathrm{d}^{3}$ ions. ${ }^{43}$

In our fitting protocol, one of the parameters was first distributed over 20 values to account for the major part of the width and asymmetry of the distribution (primary parameter) and after a satisfactory fit was obtained the other parameter (secondary parameter) was distributed along a normal curve, also of 20 values; the composite spectra of simultaneous distributions were thus the weighted sum of $20 \times 20=400$ spectra covering all pairs of the two parameters. In the absence of a microscopic model, and in order to avoid unsubstantiated hypotheses, we treat the two variables as uncorrelated, although some correlation may probably exist between them (see 
Supporting Information for more details on the statistical treatment). The results of the fits are shown in Figure 6. 

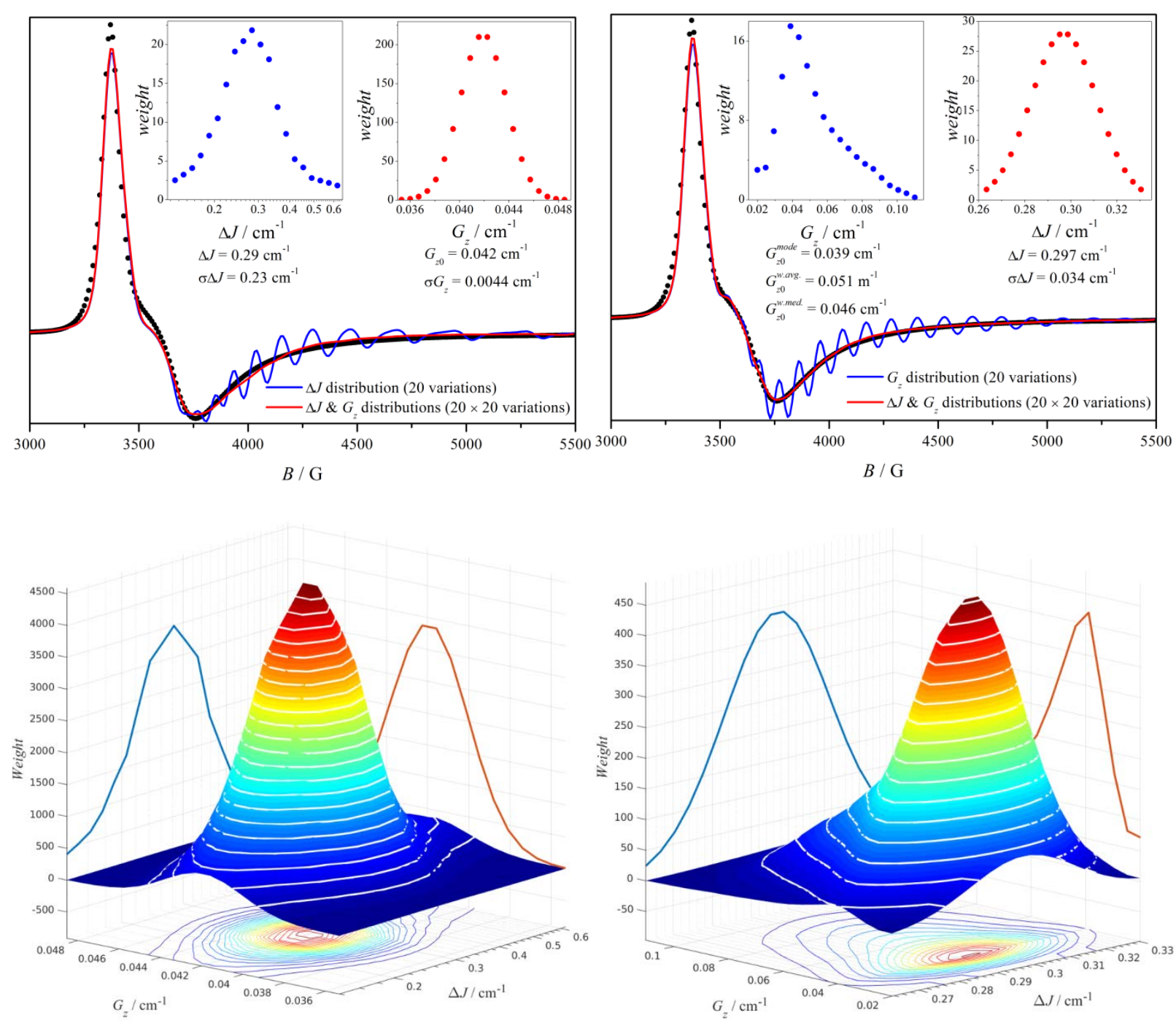

Figure 6. Top: Simulations considering an empirical distribution of the primary parameter (blue insets, blue calculated spectra) and an additional normal distribution of the secondary parameter (red insets). The red calculated spectra correspond to consideration of both distributions. Top left: $\Delta J$ as primary parameter (logarithmic scale). Top right: $G_{z}$ as primary parameter.

Bottom: 3D surface plots of the derived distributions of the $\left(\Delta J, G_{z}\right)$ pairs considering the distributions of the individual parameters. Bottom left: solution A. Bottom right: solution B. The 2D plots of the $\Delta J$ and $G_{z}$ distributions on the side walls have been scaled for clarity; the $z$ axis values correspond to the surface plots. 
To better understand how these pairs of distributions compare to each other, and to the conclusions of IINS studies, their contour plots are shown together, along with line segments that outline the limiting conditions $1.13 \mathrm{~cm}^{-1}<\Delta<2.26 \mathrm{~cm}^{-1}$ (Figure 7, dashed lines). This plot shows that solution $\mathbf{B}$ stays more within the confines of the limiting conditions, while solution $\mathbf{A}$ exceeds the lower bound for $\Delta$. Physically, this can be explained by the fact that those limits are "soft", since they correspond to the centers of Lorentzians, whose tails span the 0 to $4 \mathrm{~cm}^{-1}$ range.

The positions and shapes of these contour plots reflect our initial assumptions: they are centered around $\Delta J \sim 0.29-0.30 \mathrm{~cm}^{-1}$ and $G_{z} \sim 0.04-0.05 \mathrm{~cm}^{-1}$ and each is longer along the primary parameter and narrower along the secondary one.

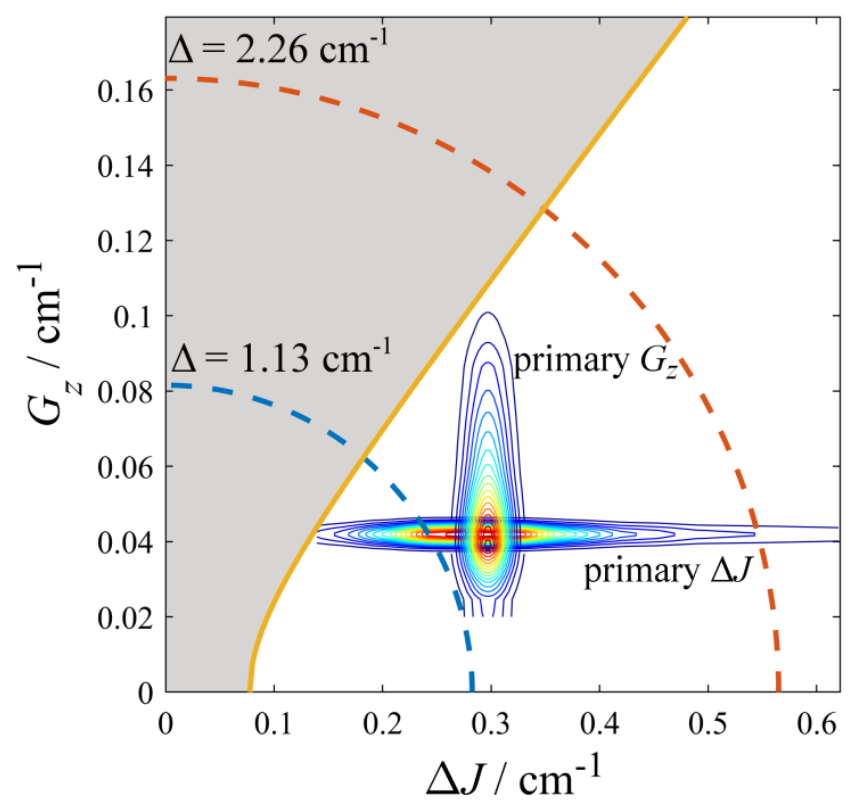

Figure 7. Contour plots of the two pairs of distributions previously determined. The blue and red dashed lines outline the IINS-determined "soft" limits indicating pairs of $\left(\Delta J, G_{z}\right)$ values that correspond to $\Delta=1.13 \mathrm{~cm}^{-1}$ and $\Delta=2.26 \mathrm{~cm}^{-1}$, respectively. The yellow solid line outlines the EPR-determined "hard" limit for which resonance conditions occur inside the experimentally observed magnetic field range $\left(f_{\mathrm{EPR}}=9.311 \mathrm{GHz}\right)$. The grey area corresponds to $\left(\Delta J, G_{z}\right)$ pairs 
that cause resonances to fall outside that window and which are discarded. For this top-right quandrant, there is also a practically symmetrical top-left one, for $\Delta J<0$ and $G_{z}>0$ forming a semicircle. The opposite semicircle would correspond to $G_{z}<0$.

\section{Discussion and conclusions}

As discussed above, we have conducted detailed simulations of the EPR spectrum of $\mathbf{1}$ by considering distributions of the effective parameters of the multispin Hamiltonian and not of the total spin (or Giant spin) Hamiltonian which acts only within the ground state multiplet. In particular, having observed the asymmetry of the distribution of $g_{\perp e f f}$, we considered two extreme cases where the asymmetry is fully absorbed by one of the two parameters (the primary one), while the other one (secondary) was normally distributed. In both cases the results are coherent regarding their mean (and median) values: in both cases $\Delta J$ is calculated around $0.29-0.30 \mathrm{~cm}^{-1}$ and $G_{z}$ around $0.04-0.05 \mathrm{~cm}^{-1}$. It is noteworthy that this value for $G_{z}$ is very close to that derived by the best fit to the magnetic susceptibility data. Both parameters are more widely distributed when they are primary than when they are secondary.

A first point that needs to be made is that these solutions are not necessarily unique, both regarding the central values and their distributions.

Regarding the central values, a different $\left(\Delta J, G_{z}\right)$ pair could be selected as a starting point for our simulations, so long as it satisfies the $1.13 \mathrm{~cm}^{-1}<\Delta<2.26 \mathrm{~cm}^{-1}$ limiting conditions. However, this limitation is not sufficient: such a starting point should not lie too close to the $G_{z}$ axis (i.e. $\Delta J \sim 0$ ) as this would imply resonances beyond the experimentally observed fields (i.e. $\sim 5500 \mathrm{G}, g \sim 1.2)$. Indeed, resonances must remain within the experimentally observed range, i.e. $g_{\| \text {eff }}>g_{\perp e f f}>1.2$. By assuming, for simplicity, that $g_{\| 0} \sim g_{\perp 0}$, for a $9.31 \mathrm{GHz}\left(0.31 \mathrm{~cm}^{-1}\right)$ 
microwave frequency, the condition $g_{\perp \text { eff }}>1.2$ can be approximately rewritten as $G_{z}<\left[\left(\Delta J^{2}-\right.\right.$ $\left.\left.6 \times 10^{-3} \mathrm{~cm}^{-2}\right) / 7\right]^{1 / 2}$. When plotted against the distributions in Figure 7, this additional limiting condition cuts the $\left(\Delta J, G_{z}\right)$ parameter space almost in half, which means that a large range of points that satisfy IINS spectroscopy lead to EPR resonances outside the observed range (grey area). So, although there is some variability for those central values, this is within narrow limits.

Moreover, for a given such point, different distributions might satisfactorily reproduce the experimental data; in our example, two such solutions were tested, in which a primary parameter was widely and asymmetrically distributed in an empirical manner, and the secondary one was more narrowly distributed on a parametrically determined symmetric curve. These models assume that the asymmetry is absorbed entirely by one parameter, while the other is symmetrically distributed. This choice, mostly imposed by computational constrains and not so much by physical considerations, may not perfectly correspond to the actual situation, but does define two $\left(\Delta J, G_{z}\right)$ limiting distributions which are realistic and physically meaningful.

A salient point of these simulations is the derivation of a lognormal distribution for $\Delta J$ in solution A. Lognormal distributions have been observed in the sizes of aerosols, ${ }^{49}$ liquid droplets in zero-gravity conditions, ${ }^{50}$ clay particles, ${ }^{51}$ various types of nanoparticles, ${ }^{52,53}$ as well as in the molecular weights of polymers. ${ }^{54}$ They have also been suggested as a general description of the sizes of magnetic dipolar couplings in solids. ${ }^{55}$ In the field of Molecular Magnetism it had been predicted that dislocations within a crystal of $\mathrm{Mn}_{12}$-acetate cause transverse anisotropy $(E)$ which is responsible for a lowering of the effective spin reversal energy barrier, and for resonant Quantum Tunneling of the Magnetization showing up for all $M_{S}$ levels within the ground $S_{\mathrm{T}}=10$ multiplet, instead of just the ones allowed for a perfect crystal of tetragonal symmetry. The experimental signature of this latter effect is the appearance of characteristic steps in the 
hysteresis cycles of $\mathrm{Mn}_{12}$ below the blocking temperature $(\sim 3 \mathrm{~K})$, at the magnetic fields where the relaxation is accelerated due to resonant QTM. These terms lift the degeneracy of the $\pm M_{S}$ levels by introducing a tunnel splitting energy $\Delta_{N}(N=0,1 \ldots S$, with $S=10)$. The initial theoretical calculations, ${ }^{56}$ subsequently confirmed by magnetization studies, ${ }^{38-40}$ indicated that the tunnel splittings are strongly transmitted over tens of unit cells around the dislocation, spanning several orders of magnitude in size and causing marked effects even for concentrations as low as 1 dislocation per 10000 molecules. Rapid thermal treatments were found to increase the mosaicity of the crystal and the tunneling rates, as they increased the density of the dislocations. ${ }^{57}$ Interestingly, these tunnel splittings were shown to exhibit a lognormal distribution.

The above observations bear great relevance to our work, at least from a phenomenological perspective, and suggest intriguing hypotheses as to the rise of these particular distributions. By drawing an analogy from the effect of crystal dislocations on the transverse anisotropies of $\mathrm{Mn}_{12-}$ acetate, we may formulate the hypothesis that similar dislocations in the crystal of $\mathbf{1}$ produce magnetic asymmetries in its magnetic couplings. An alternative interpretation is that, instead of crystal dislocations, randomly created distortions are created by disorders in the crystal lattice stemming from the disordered perchlorates and pyridine solvate molecules in the crystal lattice of 1. Moreover, the pyridine solvate molecules have half occupancies and their absences from the asymmetric units should be randomly distributed throughout the lattice. Either of those effects, i.e. disorder or half occupancies could provoke a subtle yet discernible effect qualitatively similar to that of dislocations.

Such conclusions, in the case of dislocations, were based on detailed theoretical studies which provided the microscopic framework for the interpretation of preceding and subsequent 
experimental studies, however, as we have stated from the outset, our methodology is the reverse, in the absence of such studies. It should also be stressed that our methodology rests on the phenomenological analysis of the spectra of $\mathbf{1}$, making no assumptions and drawing no conclusions regarding the static or dynamic nature of the magnetic asymmetry, a question which would require theoretical studies and time-resolved experiments to be adequately addressed.

Thus, from a quantitative perspective, we can determine with a high degree of confidence that the central value of $\Delta J$ falls within the $0.3-0.4 \mathrm{~cm}^{-1}$ range with a FWHM of $0.1-0.2 \mathrm{~cm}^{-1}$, and the central value of $G_{z}$ falls within the $0.02-0.06 \mathrm{~cm}^{-1}$ range with a FWHM of $0.01-0.03 \mathrm{~cm}^{-1}$. This determination of $G_{z}$ is several times smaller than the Moriya estimate ${ }^{58}$ of $G_{z} \sim\left|\left(g-g_{\mathrm{e}}\right) J_{\mathrm{av}} / g_{\mathrm{e}}\right|$ which gives $0.16 \mathrm{~cm}^{-1}$, suggesting that more detailed models are required to explain it. Since abnormally large values are often reported for this parameter, it should be noted that the derived value is perfectly within physically meaningful values. To put those values in perspective, $\Delta J$ is determined to $3-4 \%$ of $J_{\mathrm{av}}$ and its distribution to $1-2 \%$ of that value. In turn, $G_{z}$ is determined to $0.2-0.6 \%$ and its distribution to $0.1-0.3 \%$ of $J_{\mathrm{av}}$. These distributions are not experimental error margins, e.g. as would be statistically calculated for parameters determined from magnetometric measurements; they are the actual distributions of these parameters based on the analysis of EPR lineshapes. These determinations illustrate the unique ability of EPR spectroscopy to analyze to great precision the effective multispin Hamiltonian parameters of spin triangles. Nevertheless, we also need to stress the complementary value of SQUID and IINS studies on that regard: both are ideally suited to precisely determine $J_{\mathrm{av}}$, while neutron studies can also determine the value of $\Delta$. However, they cannot determine the character of $\Delta$, i.e. to what extent it stems from a symmetry lowering or antisymmetric exchange interactions. The power of EPR spectroscopy lies in the fact that it can reveal the interplay of those two factors. 
Finally, in comparison to its ferric analogue, magnetic studies revealed a ratio of the average isotropic couplings, $J_{\mathrm{av}}$, of $J_{\mathrm{av}}^{\mathrm{Fe}} / J_{\mathrm{av}} \mathrm{Cr} \sim 2: 1$, which was rationalized through the single-ion electronic configurations, which favour a stronger interaction in the case of $\mathrm{Fe}^{\mathrm{III}}$. Moreover, while the $G_{z} / J_{\mathrm{av}}$ and $G_{z} / \Delta J$ ratios were around $10 \%$ and $60 \%$, respectively, for the ferric analogue, they were only around $0.46 \%$ and $15 \%$ for the chromic complex $\mathbf{1}$. So, when compared to the $\mathrm{Fe}^{\mathrm{III}}$ complex, not only was the isotropic exchange found to be significantly weaker in the case of the $\mathrm{Cr}^{\mathrm{III}}$ complex, but the antisymmetric exchange was found to be $\sim 20$ times weaker in terms of $J_{\mathrm{av}}$ and 4 times weaker in terms of $\Delta J$. This much weaker antisymmetric exchange interaction may also be rationalized through the lower efficiency of the superexchange mechanism.

Concerning the strain description, both complexes were analysed by models that assumed a $J_{\mathrm{av}}$ not subject to variations or distributions; only the $J_{\text {av }}$ components, $J$ and $J^{\prime}$, were subject to such effects. However, whereas the ferric complex could be successfully analysed through a dynamic model which entails discontinuous transitions between isosceles and scalene magnetic conformations (with a $30^{\circ}$ step for the deformation vector), complex $\mathbf{1}$ had to be analysed through a model considering continuous distributions of isosceles conformations. Indeed, the EPR linewidths predicted by the dynamic model were too small to account for the experimental spectrum of $\mathbf{1}$. In other words, while the MJTE for the ferric complex could be explained without loss of the average molecular symmetry, this was not the case for $\mathbf{1}$. These findings demonstrate that while a change in metal ion does not change the general magnetic structure of spin triangles, it significantly influences their finer details, even though structural parameters remain practically unchanged. 
In conclusion, we have developed a method for the treatment of EPR data of spin triangles, which treats quantitatively the various strain effects and extracts the maximum amount of information regarding the central values and distribution profiles of their spin Hamiltonian parameters. By applying this method to complex 1, we were able to quantify to a good approximation, and in excellent agreement to SQUID and IINS data, the central values and distribution profiles of its spin Hamiltonian parameters. This treatment revealed much weaker magnetic asymmetries and antisymmetric exchange interactions when going from iron(III) to chromium(III). While the development of a microscopic mechanism for the loss of magnetic symmetry in $\mathbf{1}$ is beyond the scope of the current work, the observation of a lognormal distribution in its $\Delta J$ values is an intriguing observation that merits further exploration in conjunction with the previous observation.

\section{Acknowledgements}

We thank Dr. Jack Harrowfield (Université de Strasbourg) for helpful discussions on the preparations of chromium(III) starting materials, Dr Nathalie Kyritsakas (Université de Strasbourg) for the collection of XRD data of complex $\mathbf{1}$ and Dr. Yiannis Sanakis (INN, NCSR "Demokritos") for discussions on the EPR spectra of spin triangles. This project has received funding from the European Union's Horizon 2020 research and innovation programme under the Marie Sklodowska-Curie grant agreement No 746060. 


\section{Associated Content}

Supporting Information. Variable-temperature EPR spectra and XRD data of 1, EPR data of the starting material, and additional magnetic susceptibility and EPR simulations are available free of charge (PDF).

\section{References}

(1) Welo, L. A. Magnetic Studies on Salts, with Particular Reference to Those with Complex Ions. Lond. Edinb. Dublin Philos. Mag. J. Sci. 1928, 6 (36), 481-509.

(2) Kambe, K. On the Paramagnetic Susceptibilities of Some Polynuclear Complex Salts. J. Phys. Soc. Jpn. 1950, 5 (1), 48-51.

(3) Rudowicz, C.; Karbowiak, M. Disentangling Intricate Web of Interrelated Notions at the Interface between the Physical (Crystal Field) Hamiltonians and the Effective (Spin) Hamiltonians. Coord. Chem. Rev. 2015, 287, 28-63.

(4) Han, T.-H.; Helton, J. S.; Chu, S.; Nocera, D. G.; Rodriguez-Rivera, J. A.; Broholm, C.; Lee, Y. S. Fractionalized Excitations in the Spin-Liquid State of a Kagome-Lattice Antiferromagnet. Nature 2012, 492 (7429), 406-410.

(5) Kahn, O. Competing Spin Interactions and Degenerate Frustration for Discrete Molecular Species. Chem. Phys. Lett. 1997, 265 (1-2), 109-114.

(6) Shores, M. P.; Nytko, E. A.; Bartlett, B. M.; Nocera, D. G. A Structurally Perfect $S=1 / 2$ Kagomé Antiferromagnet. J. Am. Chem. Soc. 2005, 127 (39), 13462-13463.

(7) Okubo, S.; Nakata, R.; Ikeda, S.; Takahashi, N.; Sakurai, T.; Zhang, W.-M.; Ohta, H.; Shimokawa, T.; Sakai, T.; Okuta, K.; et al. Dzyaloshinsky-Moriya Interaction and the Ground State in $S=3 / 2$ Perfect Kagome Lattice Antiferromagnet $\mathrm{KCr}_{3}(\mathrm{OH})_{6}\left(\mathrm{SO}_{4}\right)_{2}(\mathrm{Cr}$ Jarosite) Studied by X-Band and High-Frequency ESR. J. Phys. Soc. Jpn. 2017, 86 (2), 024703.

(8) Leuenberger, M. N.; Loss, D. Quantum Computing in Molecular Magnets. Nature 2001, 410 (6830), 789-793.

(9) Meier, F.; Levy, J.; Loss, D. Quantum Computing with Antiferromagnetic Spin Clusters. Phys. Rev. B 2003, 68 (13), 134417.

(10) Mitrikas, G.; Sanakis, Y.; Raptopoulou, C. P.; Kordas, G.; Papavassiliou, G. Electron Spin-Lattice and Spin-Spin Relaxation Study of a Trinuclear Iron(III) Complex and Its Relevance in Quantum Computing. Phys Chem Chem Phys 2008, 10 (5), 743-748.

(11) Bertaina, S.; Gambarelli, S.; Mitra, T.; Tsukerblat, B.; Müller, A.; Barbara, B. Quantum Oscillations in a Molecular Magnet. Nature 2008, 453 (7192), 203-206.

(12) Lutz, P.; Marx, R.; Dengler, D.; Kromer, A.; van Slageren, J. Quantum Coherence in a Triangular $\mathrm{Cu}_{3}$ Complex. Mol. Phys. 2013, 111 (18-19), 2897-2902.

(13) Carretta, S.; Santini, P.; Amoretti, G.; Troiani, F.; Affronte, M. Spin Triangles as Optimal Units for Molecule-Based Quantum Gates. Phys. Rev. B 2007, 76 (2), 024408.

(14) Trif, M.; Troiani, F.; Stepanenko, D.; Loss, D. Spin-Electric Coupling in Molecular Magnets. Phys. Rev. Lett. 2008, 101 (21), 217201. 
(15) Trif, M.; Troiani, F.; Stepanenko, D.; Loss, D. Spin Electric Effects in Molecular Antiferromagnets. Phys. Rev. B 2010, 82 (4), 045429.

(16) Troiani, F.; Stepanenko, D.; Loss, D. Hyperfine-Induced Decoherence in Triangular SpinCluster Qubits. Phys. Rev. B 2012, 86 (16), 161409(R).

(17) Boudalis, A.; Robert, J.; Turek, P. First Demonstration of Magnetoelectric Coupling in a Polynuclear Molecular Nanomagnet: Single-Crystal EPR Studies of

$\left[\mathrm{Fe}_{3} \mathrm{O}\left(\mathrm{O}_{2} \mathrm{CPh}\right)_{6}(\mathrm{Py})_{3}\right]\left(\mathrm{ClO}_{4}\right) \cdot$ py under Static Electric Fields. Chem. - Eur. J. 2018, DOI: 10.1002/chem.201803038.

(18) DiVincenzo, D. P. The Physical Implementation of Quantum Computation. Fortschritte Phys. 2000, 48 (9-11), 771-783.

(19) Cannon, R. D.; White, R. P. Chemical and Physical Properties of Triangular Bridged Metal Complexes. In Progress in Inorganic Chemistry; Lippard, S. J., Ed.; John Wiley \& Sons, Inc.: Hoboken, NJ, USA, 2007; pp 195-298.

(20) Murao, T. Jahn-Teller Effect in Trinuclear Complexes. Phys. Lett. A 1974, 49 (1), 33-35.

(21) Uryû, N.; Friedberg, S. A. Magnetic and Thermal Properties of $\left[\mathrm{Cr}_{3}\left(\mathrm{CH}_{3} \mathrm{COO}\right)_{6}(\mathrm{OH})_{2}\right] \mathrm{Cl} \cdot 8 \mathrm{H}_{2} \mathrm{O}$, a System of Clusters of Three $\mathrm{Cr}^{3+}$ Ions. Phys. Rev. 1965, 140 (5A), A1803-A1811.

(22) Boča, R. A Handbook of Magnetochemical Formulae, 1st ed. 2012.; Elsevier insights; Elsevier: London; Waltham, MA, 2012.

(23) Mishima, M.; Uryû, N. Low Temperature Magnetic Properties of Trimeric Clusters. Phys. Lett. A 1978, 67 (1), 64-66.

(24) Rakitin, Y. V.; Yablokov, Y. V.; Zelentsov, V. V. EPR Spectra of Trigonal Clusters. J. Magn. Reson. 1969 1981, 43 (2), 288-301.

(25) Tsukerblat, B.; Belinskii, M.; Fainzil’berg, V. Magnetochemistry and Spectroscopy of Transition Metal Exchange Clusters. Sov. Sci Rev B Harwood Acad Pub 1987, 337-482.

(26) Guigliarelli, B.; Gayda, J. P.; Bertrand, P.; More, C. Relationship between Structural and Magnetic Properties of the 3Fe Clusters in Iron-Sulfur Proteins. Biochim. Biophys. Acta BBA - Protein Struct. Mol. Enzymol. 1986, 871 (2), 149-155.

(27) Guigliarelli, B.; More, C.; Bertrand, P.; Gayda, J. P. $\tilde{G}$-tensor Calculation for Fe ${ }^{\mathrm{III}}$ Triads: Application to the 3Fe Clusters in Iron-Sulfur Proteins. J. Chem. Phys. 1986, 85 (5), 2774-2778.

(28) Sanakis, Y.; Macedo, A. L.; Moura, I.; Moura, J. J. G.; Papaefthymiou, V.; Münck, E. Evidence for Antisymmetric Exchange in Cuboidal [3Fe-4S ${ }^{+}$Clusters. J. Am. Chem. Soc. 2000, 122 (48), 11855-11863.

(29) Stamatatos, T. C.; Vlahopoulou, J. C.; Sanakis, Y.; Raptopoulou, C. P.; Psycharis, V.; Boudalis, A. K.; Perlepes, S. P. Formation of the Core in Copper(II) Carboxylate Chemistry via Use of Di-2-Pyridyl Ketone Oxime $\left[(\mathrm{Py})_{2} \mathrm{CNOH}\right]:\left[\mathrm{Cu}_{3}(\mathrm{OH})\left(\mathrm{O}_{2} \mathrm{CR}\right)_{2}\left\{(\mathrm{Py})_{2} \mathrm{CNO}\right\}_{3}\right](\mathrm{R}=\mathrm{Me}, \mathrm{Ph})$. Inorg. Chem. Commun. 2006, 9 (8), 814-818.

(30) Boudalis, A. K.; Rogez, G.; Heinrich, B.; Raptis, R. G.; Turek, P. Towards Ionic Liquids with Tailored Magnetic Properties: Bmim ${ }^{+}$Salts of Ferro- and Antiferromagnetic $\mathrm{Cu}_{3}$ Triangles. Dalton Trans 2017, 46 (36), 12263-12273.

(31) Boudalis, A. K.; Sanakis, Y.; Dahan, F.; Hendrich, M.; Tuchagues, J.-P. An Octanuclear Complex Containing the $\left\{\mathrm{Fe}_{3} \mathrm{O}\right\}^{7+}$ Metal Core: Structural, Magnetic, Mössbauer, and Electron Paramagnetic Resonance Studies. Inorg. Chem. 2006, 45 (1), 443-453. 
(32) Georgopoulou, A. N.; Margiolaki, I.; Psycharis, V.; Boudalis, A. K. Dynamic versus Static Character of the Magnetic Jahn-Teller Effect: Magnetostructural Studies of $\left[\mathrm{Fe}_{3} \mathrm{O}\left(\mathrm{O}_{2} \mathrm{CPh}\right)_{6}\left(\mathrm{H}_{2} \mathrm{O}\right)_{3}\right] \mathrm{ClO}_{4} \cdot$ py. Inorg. Chem. 2017, 56 (2), 762-772.

(33) Sanakis, Y.; Boudalis, A. K.; Tuchagues, J.-P. J-Strain and Antisymmetric Exchange in a Polynuclear Compound Containing the $\left\{\mathrm{Fe}_{3} \mathrm{O}\right\}^{7+}$ Core. Comptes Rendus Chim. 2007, 10 (1-2), 116-124.

(34) Psycharis, V.; Raptopoulou, C. P.; Boudalis, A. K.; Sanakis, Y.; Fardis, M.; Diamantopoulos, G.; Papavassiliou, G. Syntheses, Structural, and Physical Studies of Basic Cr ${ }^{\mathrm{III}}$ and $\mathrm{Fe}^{\mathrm{III}}$ Benzilates and Benzoates: Evidence of Antisymmetric Exchange and Distributions of Isotropic and Antisymmetric Exchange Parameters. Eur. J. Inorg. Chem. 2006, 2006 (18), 3710-3723.

(35) Vlachos, A.; Psycharis, V.; Raptopoulou, C. P.; Lalioti, N.; Sanakis, Y.; Diamantopoulos, G.; Fardis, M.; Karayanni, M.; Papavassiliou, G.; Terzis, A. A Nearly Symmetric Trinuclear Chromium(III) Oxo Carboxylate Assembly: Preparation, Molecular and Crystal Structure, and Magnetic Properties of $\left[\mathrm{Cr}_{3} \mathrm{O}\left(\mathrm{O}_{2} \mathrm{CPh}\right)_{6}(\mathrm{MeOH})_{3}\right]\left(\mathrm{NO}_{3}\right) \cdot 2 \mathrm{MeOH}$. Inorganica Chim. Acta 2004, 357 (11), 3162-3172.

(36) Clayton, J. A.; Keller, K.; Qi, M.; Wegner, J.; Koch, V.; Hintz, H.; Godt, A.; Han, S.; Jeschke, G.; Sherwin, M. S.; et al. Quantitative Analysis of Zero-Field Splitting Parameter Distributions in Gd( III ) Complexes. Phys. Chem. Chem. Phys. 2018, 20 (15), 1047010492.

(37) Brozek, C. K.; Ozarowski, A.; Stoian, S. A.; Dincă, M. Dynamic Structural Flexibility of Fe-MOF-5 Evidenced by ${ }^{57}$ Fe Mössbauer Spectroscopy. Inorg. Chem. Front. 2017, 4 (5), $782-788$.

(38) Mertes, K. M.; Suzuki, Y.; Sarachik, M. P.; Paltiel, Y.; Shtrikman, H.; Zeldov, E.; Rumberger, E.; Hendrickson, D. N.; Christou, G. Distribution of Tunnel Splittings in Mn 12 Acetate. Phys. Rev. Lett. 2001, 87 (22).

(39) Barco, E. del; Kent, A. D.; Rumberger, E. M.; Hendrickson, D. N.; Christou, G. Tunneling Splittings in $\mathrm{Mn}_{12}$-Acetate Single Crystals. Europhys. Lett. EPL 2002, 60 (5), 768-774.

(40) Mertes, K. M.; Suzuki, Y.; Sarachik, M. P.; Myasoedov, Y.; Shtrikman, H.; Zeldov, E.; Rumberger, E. M.; Hendrickson, D. N.; Christou, G. More Evidence for a Distribution of Tunnel Splittings in Mn12-Acetate. J. Appl. Phys. 2003, 93 (10), 7095-7097.

(41) Kliava, J.; Berger, R. Size and Shape Distribution of Magnetic Nanoparticles in Disordered Systems: Computer Simulations of Superparamagnetic Resonance Spectra. $J$. Magn. Magn. Mater. 1999, 205 (2-3), 328-342.

(42) Rakitin, Y. V.; Kalinnikov, V. T.; Novotortsev, V. M. Jahn-Teller Effect in Trigonal $\mu-$ Oxoclusters. Russ. Chem. Bull. 2004, 53 (11), 2478-2484.

(43) Moskvin, A. S.; Bostrem, I. G. Some Peculiarities of Exchange Interactions in Orthoferrite-Orthochromites. Sov Phys Solid State 1977, 19, 1532-1538.

(44) Cannon, R. D.; Jayasooriya, U. A.; Sowrey, F. E.; Tilford, C.; Little, A.; Bourke, J. P.; Rogers, R. D.; Vincent, J. B.; Kearley, G. J. Concealed Asymmetry in an ExchangeCoupled Trichromium(III) Cluster: Structure and Magnetic Spectrum of $\left[\mathrm{Cr}_{3} \mathrm{O}(\mathrm{OOCPh})_{6}(\mathrm{Py})_{3}\right](\mathrm{Py})_{0.5} \mathrm{ClO}_{4}$. Inorg. Chem. 1998, 37 (21), 5675-5677.

(45) Chilton, N. F.; Anderson, R. P.; Turner, L. D.; Soncini, A.; Murray, K. S. PHI: A Powerful New Program for the Analysis of Anisotropic Monomeric and Exchange-Coupled Polynuclear $d$ - and $f$-Block Complexes. J. Comput. Chem. 2013, 34 (13), 1164-1175. 
(46) Stoll, S.; Schweiger, A. EasySpin, a Comprehensive Software Package for Spectral Simulation and Analysis in EPR. J. Magn. Reson. 2006, 178 (1), 42-55.

(47) Sowrey, F. E.; Tilford, C.; Wocadlo, S.; Anson, C. E.; Powell, A. K.; Bennington, S. M.; Montfrooij, W.; Jayasooriya, U. A.; Cannon, R. D. Spin Frustration and Concealed Asymmetry: Structure and Magnetic Spectrum of $\left[\mathrm{Fe}_{3} \mathrm{O}\left(\mathrm{O}_{2} \mathrm{CPh}\right)_{6}(\mathrm{Py})_{3}\right] \mathrm{ClO}_{4} \cdot \mathrm{py}$. J. Chem. Soc. Dalton Trans. 2001, No. 6, 862-866.

(48) Boča, R. Zero-Field Splitting in Metal Complexes. Coord. Chem. Rev. 2004, 248 (9-10), 757-815.

(49) Aleksandropoulou, V.; Eleftheriadis, K.; Diapouli, E.; Torseth, K.; Lazaridis, M. Assessing $\mathrm{PM}_{10}$ Source Reduction in Urban Agglomerations for Air Quality Compliance. J Env. Monit 2012, 14 (1), 266-278.

(50) Oprisan, A.; Garrabos, Y.; Lecoutre, C.; Beysens, D. Pattern Evolution during Double Liquid-Vapor Phase Transitions under Weightlessness. Molecules 2017, 22 (6), 947.

(51) Tan, X.; Liu, F.; Hu, L.; Reed, A. H.; Furukawa, Y.; Zhang, G. Evaluation of the Particle Sizes of Four Clay Minerals. Appl. Clay Sci. 2017, 135, 313-324.

(52) Monteil, A.; Ghemid, S.; Chaussedent, S.; El Jouad, M.; Couto dos Santos, M. A. Molecular Dynamics Simulation of Silver Nanoparticles in a Europium Doped Sodosilicate Glass. Chem. Phys. Lett. 2010, 493 (1-3), 118-120.

(53) Kanhe, N. S.; Kumar, A.; Yusuf, S. M.; Nawale, A. B.; Gaikwad, S. S.; Raut, S. A.; Bhoraskar, S. V.; Wu, S. Y.; Das, A. K.; Mathe, V. L. Investigation of Structural and Magnetic Properties of Thermal Plasma-Synthesized $\mathrm{Fe}_{1-\mathrm{x}} \mathrm{Ni}_{\mathrm{x}}$ Alloy Nanoparticles. $J$. Alloys Compd. 2016, 663, 30-40.

(54) Cao, Q.; Tian, P.; Wu, Q. Modeling Diameter Distributions of Poly $(N-$ Isopropylacrylamide-Co-Methacrylic Acid) Nanoparticles. J. Appl. Polym. Sci. 2009, 111 (5), 2584-2589.

(55) Lacelle, S.; Tremblay, L. What Is a Typical Dipolar Coupling Constant in a Solid? J. Chem. Phys. 1993, 98 (5), 3642-3649.

(56) Chudnovsky, E. M.; Garanin, D. A. Spin Tunneling via Dislocations in Mn 12 Acetate Crystals. Phys. Rev. Lett. 2001, 87 (18).

(57) Hernandez, J. M.; Torres, F.; Tejada, J.; Molins, E. Crystal Defects and Spin Tunneling in Single Crystals of Mn 12 Clusters. Phys. Rev. B 2002, 66 (16).

(58) Moriya, T. Anisotropic Superexchange Interaction and Weak Ferromagnetism. Phys. Rev. 1960, 120 (1), 91-98. 


\section{Table of contents entry}

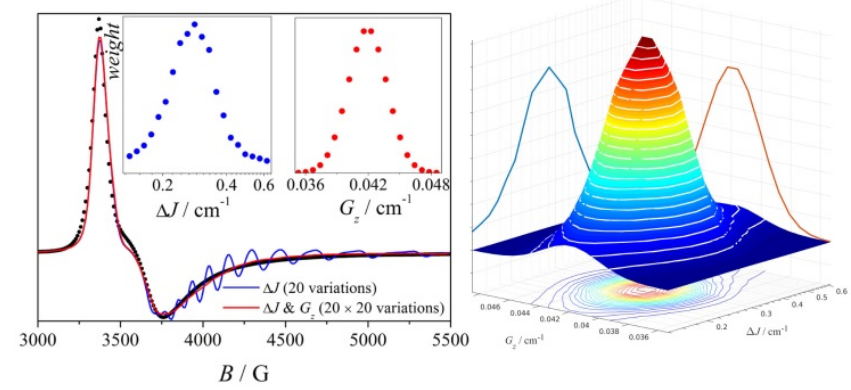

The EPR spectrum of a highly symmetric chromium(III) spin triangle has revealed highly anisotropic and broadened features. These have been analyzed by full Hamiltonian methods, which consider simultaneous distributions of its isotropic and antisymmetric exchange (Dzyaloshinskii-Moriya) interaction parameters. Examination of several broadening models has allowed the determination of the central values and distribution profiles of these parameters. 